\title{
A Survey of Nurses' Knowledge Regarding Prevention and Management of Diabetic Foot Ulcer in Bangladesh
}

\author{
SHARMISTHA $S^{\mathrm{a}}$, WONGCHAN $\mathrm{P}^{\mathrm{b}}$, HATHAIRAT S ${ }^{\mathrm{c}}$
}

\begin{abstract}
Diabetic foot ulcer is a preventable complication of diabetes. Nurses must have knowledge related to this condition so that education can be given to patients. This study aims to examine the level of nurses' knowledge regarding prevention and management of diabetic foot ulcer in Bangladesh. A survey involved 218 nurses working at a national, specialized, and well-established institute of diabetic care of Bangladesh. The Nurses' Knowledge Regarding Prevention and Management of Diabetic Foot Ulcer Questionnaire (NKPMDFUQ) comprising of 40 questions with 20 true/false questions and 20 multiple choice questions was used. It was content validated and tested for internal consistency and
\end{abstract}

\section{Introduction}

Diabetic foot ulcer (DFU) is a known-devastating complication of diabetes. Approximately $15 \%$ of people with diabetes will experience a foot ulcer in their life time1. The incidence is increasing throughout the globe. In the developed countries such as the Netherlands, United Kingdom, and Sweden, the annual incidence was $2.1 \%, 2.2 \%$, and $3.6 \%$, respectively and a 3 -year incidence in the United States was $5.8 \% .^{2}$ In Bangladesh, a retrospective cohort study conducted by Samira, Sayeed, Lahiry, and Ali (as cited in Paul and colleagues' work) ${ }^{3}$ in a diabetic hospital, the BIRDEM (Bangladesh Institute of Research Rehabilitation in Diabetes, Endocrine and Metabolic Disorders), found that the prevalence of DFU was $2.8 \%$.

a. Sharmistha Shil, RN, MN National Institute of Cardiovascular Diseases and Hospital (NICVD)

b. Wongchan Petpichetchian, RN, PhD. Faculty of Nursing, Prince of Songkla University, Thailand

c. Hathairat Sangchan, RN, PhD. Faculty of Nursing, Prince of Songkla University, Thailand

Address of Correspondence : Dr. Shil, Sharmistha Shila_shil54 @yahoo.com 1/26, Babor Road, Mohammadpur, Dhaka. Mob- 01712608713

Received: November 15, 2012

Accepted: July 09, 2013 stability yielding acceptable levels of reliability. The findings revealed that nurses had very low level of knowledge ( $M=52.60 \%, S D=7.86 \%)$. The content areas most nurses did not have knowledge were detecting loss of protective sensation of the feet, caring of callus formation, encouraging patients to have activity of daily living in order to self-manage, setting a goal for prevention of amputation, and giving advice to patients that causes of diabetes is the least important element in diabetic foot care program. The findings are beneficial for establishing training courses. Consequently, quality of care for patients with diabetes will be improved.

Keywords: Nurses' knowledge, diabetic foot ulcer

(Birdem Med J 2014; 4(1): 22-26)

DFU adds to functional disability, mental stress to the patients, and family burden. In some cases, disabled condition leads to decreased quality of life and high mortality risk. ${ }^{4}$ Bradbury and Price ${ }^{5}$ reported from their cross-sectional, exploratory study that patients with diabetic foot ulcers also had experienced physical and psychological effects. They reported some degree of DFU pain, feeling tired/exhausted requiring their efforts in coping with the situation.

As DFU is preventable, it is crucial for all health care providers including nurses to be able to prevent an ulcer before it develops. If patients already come with ulcers, nurses must be able to manage DFU to prevent further severe complications, such as wound infection and necrosis that may end up in amputation or hasten death. Nurses must be competent as demonstrated by having accurate and comprehensive knowledge as well as ability to perform necessary measures to screen patients who are at risk so that preventive measures and risk management can be executed. ${ }^{6}$ They also should be able to manage DFU properly and promptly once it develops. Unfortunately, nurses may not be prepared to possess these competencies. 
Livingston and Dunning ${ }^{7}$ reported that basic nursing education in Australia did not adequately prepare practicing nurses to meet the demands of the expected roles. McIntosh and Ousey ${ }^{8}$ conducted a survey in UK with nurses and podiatrist participants prior to giving a one-day education in wound care. Certain areas of diabetic foot care were assessed. The variation and inconsistent practices across the participants could impact patient care and could result in patients receiving suboptimal treatments. Shiu and Wong ${ }^{9}$ conducted a small-scale survey specifically on 65 registered nurses (RNs) in Hong Kong. They found that RNs who had received prior training had diabetes foot care knowledge scores higher than those who did not receive.

To date, there is no known study to examine Bangladeshi nurse's knowledge in the prevention and management of DFU. Therefore, the extent to which nurses possess this knowledge is still unidentified. In Bangladesh the majority of nurses earn diploma in nursing and some earn bachelor in nursing. Both levels of nursing education offer very limited knowledge, especially on foot care of patients having diabetes. Nurses gain practical knowledge through their daily work where the ratio of nurses to patients is considered high. ${ }^{11,12}$ Therefore, they actually have little or no time to discuss with doctors for gaining more knowledge. ${ }^{13}$ Information about standard nursing practice guidelines in the prevention of DFU that can be used as a guide in nursing practice is also lacking. For this reason, the researchers are interested in surveying nurses' knowledge regarding prevention and management of diabetic foot ulcer in Bangladesh.

\section{Materials and Methods}

A descriptive-survey study was conducted. An approval was obtained from the Research Ethics Committee of the Faculty of Nursing, Prince of Songkla University, Thailand. The further approval was also acquired from the Review Teams and the Directors of BIRDEM hospital, Dhaka, Bangladesh. Using simple random sampling, 218 nurses who had a minimum of diploma degree in nursing and had at least three months of working experience in the target hospital were recruited. There were two questionnaires used in this study. First, the demographic data questionnaire asked subjects about their age, gender, religion, level of nursing education, marital status, short course training on foot ulcer care or diabetic foot care, current area of practice, and duration of practice with patients with DM and whether they had taken care of patients with diabetic foot ulcers.

Second, the NKPM-DFU questionnaire was used to examine the level of nurses' knowledge regarding prevention and management of DFU. The questionnaire was designed using the (Registered Nurses' Association of Ontario) RNAO's structure of recommendations for assessment and management of DFU in integration with related literature on diabetes, prevention and risk management of DFU. The NKPM-DFUQ had six components composing of (1) patient empowerment and education, (2) holistic assessment, (3) foot ulcer assessment, (4) identifying goals of care, (5) management of systemic, local and extrinsic factors and (6) evaluation. It comprised of 40 items divided into two sets. In the first set, twenty true-false items were constructed. If the subjects did not know the answer, they selected “Don't Know" option. The second set comprised of twenty multiple-choice items with 5 options for each item. The correct answer of each item was scored " 1 ", and “0” for incorrect and "Don't Know" responses. The total score ranged from 0 to 40 and were transformed to percentage. Higher scores indicated the higher level of knowledge. For easy interpretation, the transformed scores were categorized into the following five categories using a general educational standard: very low (<60.00\%), low (60.00-69.99\%), moderate (70.00$79.99 \%)$, high (80.00-89.99\%) and very high (90.00100.00\%).

The NKPM-DFUQ was back translated into the Bangla language in this study. The content validity of the questionnaire was validated by three experts who were experts in diabetic care. The reliability was tested for internal consistency reliability, using KR-20 coefficient, and the test-retest for stability reliability using intraclass correlation coefficient (ICC) with a 7-day interval, yielding the coefficients of 0.64 and 0.78 , respectively. Results A total of 218 nurses had age ranged from 23 to 53 years with a mean age of 32 years old. Table 1 shows that the majority of them were female (96.3\%) and married (82.6\%). More than half of them were Muslim (60.1\%). Most of them (97.2\%) completed diploma degree and only one subject completed master degree. All subjects reported that they had never received any training regarding prevention and management of diabetic foot ulcer. Surgical (33.9\%) and medical (25.2\%) areas of 
practice comprised three-fifths of the subjects. The average years of duration of practice was 8.03(SD = 5.82), ranging from 1-27 years. The majority of them indicated having taken care of patients with diabetic foot ulcer (98.2\%).

Table-I

Nurses’ Demographic Characteristics $(N=218)$

\begin{tabular}{lcc} 
Characteristics & $\mathrm{n}$ & $\%$ \\
\hline Gender: Female & 210 & 96.3 \\
Religion: Islam & 131 & 60.1 \\
Level of nursing education: & & \\
Diploma in nursing & 212 & 97.2 \\
Marital status: Married & 180 & 82.6 \\
Short course training: No & 218 & 100 \\
Area of practice: & & \\
Others (CCU,ICU, Urology) & 77 & 35.3 \\
Surgical & 74 & 33.9 \\
Medical & 55 & 25.3 \\
Orthopedic & 12 & 5.5 \\
Care of DFU: Yes & 214 & 98.2 \\
\hline
\end{tabular}

When categorizing the nurses according to their level of knowledge, it was found that only four nurses (1.9\%) had the total knowledge scores $70 \%$ and above. The total nurses' knowledge regarding prevention and management of DFU and its subscale scores are shown in Table-II.

\section{Table-II}

Frequency and Percentage of Nurses' Classified According to the Level of Nurses' Knowledge Regarding Prevention and Management of DFU Scores $(N=218)$

\begin{tabular}{lcc} 
Level of Knowledge (Total score \%) & $\mathrm{n}$ & $\%$ \\
\hline Very low $(<60 \%)$ & 180 & 82.5 \\
Low $(60-69.99 \%)$ & 34 & 15.6 \\
Moderate (70-79.99\%) & 3 & 1.4 \\
High (80-89.99\%) & 1 & 0.5 \\
Very high (90-100\%) & 0 & 0 \\
\hline
\end{tabular}

Further item analysis was made to find out which items more nurses could answer correctly. The five items with highest and lowest percentage of nurses answered correctly are presented in Table-III.

Table-III

Five Items with Highest and Lowest Percentage of Nurses Answered Correctly on the Nurses'

Knowledge Regarding Prevention and Management of Diabetic foot Ulcer Questionnaire (NKPM-DFUQ)

\begin{tabular}{lll} 
Item Statements & $\mathrm{n}$ & $\%$ \\
\hline
\end{tabular}

Highest Five Items

1. $\quad$ Empowering and reassuring patients 21699.6 to have routine foot care

2. $\quad$ Educating patients to control blood 21598.6 sugar level (Keeping fasting blood sugar $<125 \mathrm{mg} / \mathrm{dl}$ or $<125 \mathrm{mg} \%$ or $<6.9 \mathrm{mmol} / \mathrm{L}$ )

3. Further investigating if the wound is 21498.2 infected

4. $\quad$ Promptly treating wound infection $\quad 211 \quad 96.8$ to prevent amputation

5. Having family members involved in $210 \quad 96.3$ diabetic foot care

Lowest Five Items

1. Using of Semmes-Weinstein $2 \quad 0.9$ Monofilament to detect loss of protective sensation of the feet

2. Caring of callus formation to prevent $2 \quad 0.9$ diabetic foot ulcer

3. $\quad$ Encouraging patients to have activity $8 \quad 3.7$ of daily living in order to self manage their foot ulcer

4. Setting a goal for prevention of $\quad 22 \quad 10.1$ amputation once the DFU is healed

$\begin{array}{llll}\text { 5. } & \text { Giving advice to patients that causes } & 27 & 12.4\end{array}$ of diabetes is less important in foot care program

\section{Discussion}

The majority of nurse subjects were female which not only was comparable to Basak's and Islam's studies but also to studies in other countries such as the UK8. From the study subjects' religious backgrounds, more than half of them were Muslim (60.1\%), which is consistent with the Bangladesh context. In addition, there was no any subjects in this study who had received or attended any courses or training programs related to prevention 
and management of diabetic foot ulcer. This finding was similar to the study of Basak14 who found that Bangladeshi nurses had inadequate knowledge regarding post-operative pain management. There was no subject in her study who had received any course or training program related to post-operative pain management.

The findings showed that the nurses who participated in this study had a very low level of knowledge regarding prevention and management of diabetic foot ulcer, presenting by the mean score of $52.60 \%$. Moreover, this very low level of knowledge was attributed to all six components of the nurses' knowledge for prevention and management of DFU, except one component: patient empowerment and education. Several factors might contribute to the very low level of knowledge regarding prevention and management of DFU among nurses in this study. Firstly, the majority of them (97.2\%) had nursing education at diploma level, and that they were not provided to have specialized knowledge nor were expected to provide such specialized care, such as prevention and management of DFU. These findings were generally similar to Basak's study ${ }^{14}$, mentioned earlier. In addition, the focus on evidence-based practice and knowledge regarding prevention and management of DFU has not been implemented, although this novel concept, evidence-based practice, has been recently integrated in a newly offered bachelor of science in nursing curriculum.

Secondly, duration of practice might affect the very low level of knowledge among nurses. However, this was not observed in this present study. Additional analysis and findings did not support this explanation. Unlike other studies, Vij, Williamson, and Gupta 15 found that nurses who had more years of working experience had knowledge level of infection control higher than that of those who had less years of experience. Lui, So, and Fong16 also revealed that nurses who had more working experience had better pain management performance compared to those who had less year of experience.

Thirdly, lack of problem-oriented nursing care may contribute to the level of nurses' knowledge. According to Hadley and Roques's study ${ }^{11}$, the researchers found that nurses spent only approximately $5.3 \%$ of their time in providing direct patient care. Finally, even though this hospital provides special services for patients with diabetes mellitus, the service is still general. Neither is there any special care offered nor use of assessment tools for assessing diabetic neuropathy. Furthermore, BIRDEM hospital is a research centre mainly led by physicians, not by nurses. In other words, nurses are not involved in research or any policy decision making. For this reason, poor knowledge in special care and research might be responsible for the low level of knowledge among nurses.

Consideration was given to each item of nurses' knowledge regarding prevention and management of DFU. The data showed that nurses had high level of knowledge regarding prevention and management of DFU in the areas of empowering and reassuring patients to have routine foot care, educating them to control blood sugar level, further investigating if the wound is infected, promptly treating wound infection to prevent amputation, and having family members involved in diabetic foot care. These data indicated that nurses had knowledge of DFU in prevention and management of this area. Nurses gained this general knowledge from their DFU prevention and management information from diploma or bachelor nursing program or service experience.

In contrast, low level of nurses' knowledge was more obviously seen when item analysis was conducted. There were two items that only two subjects could answer correctly. These items were: "using of SemmesWeinstein Monofilament to detect loss of protective sensation of the feet," and "caring of callus formation to prevent diabetic foot ulcer." This knowledge was highly specialized and nurses might not be educated or even observe in their real practice settings.

This study was the first survey study in Bangladesh which explored nurses' knowledge regarding prevention and management of DFU that offered good evidence to support further initiation for an improvement of nurses' competencies in this specialized care area. Yet, the findings must be viewed with caution due to some identified limitations. Similar to other survey studies, some constraints due to self-report type of data collection may exist. Although the researchers allowed a 7-day period for each subject to complete and return the questionnaires, some of them might do it hurriedly or share their answers. Moreover, the NKPM-DFUQ developed for use in this study was considered new 
and requires further investigation to examine whether its proposed structures would be confirmed. Confirmatory factor analysis and a replication study at other settings are recommended.

\section{Conclusion}

This descriptive study employed 218 nurses working at a specialized diabetes hospital, located in Dhaka city of Bangladesh. This study revealed that nurses possessed a very low level of knowledge regarding prevention and management of DFU. Possible reasons for these findings may be due to lack of update knowledge and no formal training on prevention and management of diabetic foot ulcer. In this regard, nurses should be more active in improving their clinical knowledge through support from medical staff. Nurses also need further continuing education and training program. Specialized care for prevention and management for DFU should be included in the training courses. For examples, they should be trained to have knowledge and skill regarding detection of sensory loss of the feet and care of callus formation, or at least they must be trained to be able to recognize when callus formation occurs so that consultation can be made promptly. This can be done as an in-service training course or as a continuing education program. Furthermore, in order to teach the student nurses, nurse educators should also be trained so that they can teach their students. Once nurses possess adequate knowledge, they will be able to teach and coach their patients with diabetes to prevent and manage this problem more effectively.

\section{Acknowledgements}

The researchers would like to express their gratitude to Faculty of Nursing and Graduate School, Prince of Songkla University in Thailand for providing academic resources and partial support for this study and to Bangladesh Government for providing a study scholarship to the first author. Gratitude is also expressed towards all study subjects and the authorities of the study hospital for their valuable contribution.

\section{References}

1. American Diabetes Association. Consensus development conference on diabetic foot wound care. Diabetes Care. 1999; 22(8): 1354-1360.

2. Jeffcoate WJ, Harding KG. Diabetic foot ulcer. Lancet. 2003;361(9368):1545-1551.

3. Paul S, Barai L, Jahan A, Haq A. A bacteriological study of diabetic foot infection in an urban tertiary care hospital of Dhaka city. Ibrahim Medical College Journal 2009;3(2):50-54

4. Ritchie L, \& Prentice, D. An exploration of nurses' perceptions regarding the implementation of a best practice guideline on the assessment and management of foot ulcers for people with diabetes. Applied Nursing Research, 2011;24:88-93.

5. Bradbury S, Price P. The impact of diabetic foot ulcer pain on patient quality of life. Wounds UK, 2011;7(4):3249.

6. Registered Nurses' Association of Ontario [RNAO]. Assessment and management of foot ulcers for people with diabetes. 2005; Available from http:// healthsci.queensu.ca/ assets/NSG_Simlab/Old_Assets/ 536_bpg_assessment_foot_ulcer__1.pdf.

7. Livingston R, Dunning R. Practices nurses' role and knowledge about diabetes management within rural and remote Australian general practices. European Diabetes Nursing. 2010;7(2):55-61.

8. McIntosh C, Ousey K. A survey of nurses' and podiatrists' attitudes, skills and knowledge of lower extremity wound care. Wounds UK. 2008;4(1):59-68.

9. Shiu ATY, Wong RYM. Diabetes foot care knowledge: A survey registered nurses. Journal of Clinical Nursing. 2011;20(15-16):2367-2370. 10. Habib SH, Biswas KB, Akter S, Sahao S, L. A. Cost-effectiveness analysis of medical intervention in patients with early detection of diabetic foot in a tertiary care hospital in Bangladesh. Journal of Diabetes and Its Complications. 2010;24(4):259-264.

11. Hadley MB, Roques A. Nursing in Bangladesh: Rhetoric and reality. Social Science and Medicine. 2007;64(6):1153-1165.

12. Oulton J, Hickey B. Review of the nursing crisis in Bangladesh, India, Nepal and Pakistan. 2009; Available from URL: http://www.unfpa.org/ sowmy/resources/docs/ libri.

13. Cockcroft A, Milne D, Oelofsen M, Karim E, Anderson N. Health services reform in Bangladesh: Hearing the views of health workers and their professional bodies. BMC Health services Research. 2011;11(2):S2-S8.

14. Basak S. Knowledge and attitudes of nurses and their practices regarding post-operative pain management in Bangladesh [Master]: Adult Nursing, Prince of Songkla University; 2010.

15. Vij A, Williamson SN, Gupta S. Knowledge and practice of nursing staff towards infection control measures in a tertiary care hospital. Journal of the Academy of Hospital Administration. 2001;13(2):7-12.

16. Lui L, So W, Fong D. Knowledge and attitudes regarding pain management among nurses in Hong Kong medical units. Journal of Clinical Nursing 2008;17(15):2014-2021. 